\title{
Filmotecas y archivos fílmicos en línea: producción, difusión, interconexión y posicionamiento en Internet
}

\author{
Alfonso López Yepes \\ Universidad Complutense de Madrid (España)
}

\section{Resumen}

Se analiza la situación actual que presentan filmotecas y archivos fílmicos en la sociedad de la información en cuanto a producción y difusión informativa de sus fondos documentales, así como su posicionamiento en Internet. Asimismo, se plantea una doble propuesta: la posibilidad de situar la mayor cantidad posible de fondos fílmicos en red y la de interconectar las instituciones que mantienen dichos fondos, con el consiguiente aprovechamiento de todos ellos de forma colaborativa y distribuida. Se ejemplifica con un proyecto universitario actualmente en ejecución sobre documentos cinematográficos de un director español depositados en una filmoteca nacional y su progresiva publicación en red. Por otra parte, en cumplimiento de los objetivos propuestos en el trabajo, se analiza el estado actual de la cuestión en relación con la documentación cinematográfica y las instituciones que se ocupan de la conservación y recuperación del cine, se teoriza al respecto y se plantea y revisa una problemática de carácter general que afecta al patrimonio fílmico nacional y a sus relaciones con el internacional. Como consecuencia de todo ello, se hace referencia a los sitios en Internet que mantienen filmotecas y archivos fílmicos españoles, con alguna mención a instituciones fílmicas internacionales y a su visibilidad y usabilidad.

Palabras clave: Filmotecas digitales. Archivos fílmicos digitales. Bibliotecas digitales. Planificación y diseño. Posicionamiento.

\section{Abstract}

This paper is focused on the current situation of moving image archives in the frame of the information society from the point of view of the production and diffusion of their documentary stocks, as well as of its positioning on the Internet. Two suggestions are made: placing the most part of filmic resources online and interconnecting institutions holding those resources, which would promote the usage of filmic documents in a collaborative and distributed way. We exemplify this by showing an I+D universitary project currently under development. This 
project deals with the progressive online publishing of the films of a single director owned by a public film arhival institution. In fulfilment of the proposed objectives, we analyze the current state of art in relation to cinematographic documentation and the institutions that take care of the conservation and recovery of the cinema. We introduce theoretical aspects on the matter and consider this task as a problem involving general issues such as national film heritage and its relation with the international framework. As a result of all this, different Internet sites holding movie archives both in Spain and abroad are reviewed, stressing visibility and usability factors.

Keywords: Digital film archives. Digital libraries. Planning and design. Positioning.

\section{Sociedad-red: estado de la cuestión}

Sociedad de la información, sociedad del conocimiento, sociedad del talento, sociedad multimedia, sociedad documental, sociedad-red...: puede seguir afirmándose, en 2006, que las nuevas tecnologías de la información continúan provocando un cambio profundo en los modos de trabajo de cualquier tipo de profesional y en cualquier labor que se realice en la ya denominada sociedad-red. Se ha producido una transformación social y cultural que ha originado un nuevo ámbito industrial y unas nuevas formas culturales, de relación y de intercomunicación personal y grupal. La mencionada transformación afecta todavía a todas las esferas profesionales y a sus variados campos de aplicación, y muy especialmente al ámbito de los archivos, bibliotecas y centros y servicios de información y documentación. Y, en consecuencia, a las filmotecas y archivos fílmicos.

La presente sociedad de la información, considerada como nuevo medio de comunicación, plantea una serie de cuestiones actuales sobre la influencia de las nuevas tecnologías de la información en el entorno del individuo y supone una reflexión sobre la transformación de nuestra vida cotidiana y de las formas de organización social. El debate sobre el futuro inmediato que están propugnando constantemente las nuevas tecnologías multimedia en la era digital permanece abierto. Por otra parte, el desarrollo imparable e incuestionable de la microinformática, de las memorias ópticas de almacenamiento y de las redes de comunicación deberá seguir complementándose permanentemente con nuevos sistemas, más inteligentes, de almacenamiento holográfico, de compresión y de producción de informaciones, de mejor calidad en la visualización, lectura y audición, de mayor velocidad en el acceso a los datos. De todo ello se están haciendo eco constantemente los numerosos foros y ámbitos especializados, como por ejemplo el CEBIT de Hannover, en cuya última edición (marzo 2006) se ha destacado especialmente el avance experimentado en el ancho de banda de las redes, la evolución de los or-

Scire. 14 : 2 (jul.-dic. 2008) 41-64. ISSN 1135-3716. 
denadores portátiles (ultraportátiles) y el alza en las capacidades de almacenamiento en función de los nuevos formatos y sistemas desarrollados.

Porque la demanda de información es tan inmensa, la generación de documentación tan ilimitada, que el sistema tradicional, basado en soportes impresos, es incapaz ya hace tiempo de controlar tal cúmulo de datos. Asimismo, la consulta, producción, almacenamiento, recuperación y difusión eminentemente digital de información, así como el uso de las redes que posibilitan el acceso y gestión virtual de la documentación de cualquier ámbito profesional, son ya hechos habituales, lo que ha provocado la aparición de una sociedad documental. En ella se generan, comunican y localizan documentos en entornos digitales, que posibilitan de forma también ya prácticamente generalizada el acceso a informaciones con contenidos multimedia (texto, imagen fija, audio y vídeo). De ahí que se utilice igualmente el término sociedad multimedia, como consecuencia de esa proliferación de documentos multiformes. La tendencia, por tanto, es, y más que nunca, hacia la distribución colaborativa del conocimiento en el marco de la sociedad del conocimiento (también denominada por algunos autores sociedad del talento).

En suma, las características de la actual sociedad de la información en estos momentos podrían resumirse, desde nuestro punto de vista, en los siguientes cuatro puntos de índole general:

1. Los efectos del actual cambio social, tecnológico y cultural sobre los hábitos de la población se manifiestan especialmente en el ámbito de los medios de comunicación y la cultura en general. Consecuencia: sociedad de la información, sociedad multimedia, sociedad-red, sociedad del conocimiento, sociedad del talento, sociedad de la innovación...

2. La tendencia a la realización de productos culturales de mejor calidad, la creación de canales temáticos y la relevante posición de portales y comunidades virtuales de usuarios sobre contenidos informativos diversos. Resultado: plataformas, portales, canales, comunidades virtuales de usuarios, foros, listas de distribución o discusión, intercomunicación profesional entre expertos...

3. Los cambios, la evolución permanente, exigen una perspectiva multidisciplinar de amplio espectro aplicada entre otros ámbitos a la producción audiovisual, la gestión cultural, la industria cultural... en un contexto global. Consecuencia: equipos de investigación multidisciplinares, trabajo en equipo, producción nacional...

4. La oferta de contenidos y el consumo de los mismos intensifica el papel de la tecnología y la convergencia de medios como catalizadores que provocan reacciones. Resultado: producción y difusión cultural apoyada en plataformas diversas con estructura digital intercomunicada, lo que asegura 
su visibilidad y multiplica la facilidad de acceso a la información, el conocimiento y el entretenimiento distribuidos...

A ello hay que añadir las tendencias (ampliamente debatidas en los sucesivos Online Information Meetings desde 1999, así como en otras ferias y foros especializados) que se están originando y desarrollando en esta primera década del siglo XXI, y que podrían apuntarse en los siguientes términos:

Servidores centralizados, ultraportátiles, "nuevas" tecnologías y "nиеvos" conceptos

- Sincronización y almacenamiento de datos: ordenadores portátiles (ultraportátiles) cada vez más ligeros $(1,9 \mathrm{~kg})$ y con mayor autonomía, y nuevos formatos y sistemas de almacenamiento (Blue-Ray Disc y HD-DVD, presentados en el CEBIT2006 de Hannover, para películas en alta definición y lectores). Mayor capacidad de almacenamiento, que crece a un ritmo de 390 gigabytes de datos cada segundo en el mundo.

- Servidores centralizados para la conexión de la sede de las empresas con sus trabajadores remotos, conectados mediante enlaces de banda ancha.

- "Nuevas" tecnologías y "nuevos" conceptos: RSS, blogs, open access, open sour$c e$, alertas, personalización, radio personalizada en Internet (Podcast), etcétera, forman parte de esta "nueva" realidad. En suma: tecnología de código abierto, estándares abiertos, distribución del conocimiento, información colaborativa, participación de los usuarios, desarrollo en comunidad, mayor interactividad..., "redes sociales" y movilidad.

\section{El imperio de Internet}

- La base de todo se sitúa en la configuración intranet/extranet/Internet, pública y privada: establecimiento de redes corporativas generalizadas y de conocimiento distribuido (se integra asimismo el conocimiento científico mediante la recuperación vía web de, por ejemplo, miles de tesis doctorales y revistas científicas a texto completo: en febrero de 2006 se pueden consultar más de 3000 tesis doctorales defendidas en 14 universidades españolas y unas 19500 revistas científicas en la Universidad Complutense).

- Redes más "inteligentes" que nunca en función del ancho de banda ilimitado (CEBIT2006, de nuevo), de forma que la inteligencia está cada vez más personalizada en el usuario final. Ofertas de ADSL con más velocidad y llamadas gratuitas: las zonas urbanas españolas concentran el $80 \%$ de las conexiones. Banda ancha en Bluetooth en 2007: velocidad de transmisión a 100 Mbps (megabits por segundo) para envío de audio y vídeo de alta definición. (La Unión Internacional de Telecomunicaciones - UIT aprobaba en 2005 un sistema de acceso a banda ancha a través de hilo telefónico de cobre hasta diez veces más rápido que el ADSL: el sistema se llama VDSL2 y permite alcanzar hasta 100 megabits por segundo en ambos sentidos a una distancia de unos 100 metros desde la central del operador hasta el cliente). 
- Web 2.0: interactividad, red de redes de intercambio e interacción y comunicación más que nunca; nuevo entorno donde las comunicaciones y el "compartir" se convierten en realidad y que sin duda va a tener efecto — ya lo está teniendo- en el ámbito social y en el económico.

\section{Un mundo inalámbrico: WI-FI}

- De fijo a móvil: convergencia de texto, imagen fija, voz, vídeo, televisión... La información multimedia (texto, cine, radio, televisión) ya se empieza a descargar en portátiles inalámbricos de banda ancha y en los propios teléfonos móviles.

- Reuniones virtuales globales.

- Expansión de la tecnología Java en la difusión de la telefonía inalámbrica: de los 3500 millones de aparatos en los que esta tecnología está presente, 1500 millones son móviles (Sun Microsystems, empresa inventora de Java: datos extraídos de Expo-Java2006).

El tamaño de la Web: algunos datos estadísticos

- 70000-90000 terabytes

Crecimiento de la Web: $30 \%$ anual

— 4000 millones de páginas

7 millones de páginas nuevas por día

220 idiomas

95\% públicamente accesible

La vida media de una página es de 44 días

\section{Profesión y trabajo cinematográficos: documentación e investigación}

En plena era digital se hace constantemente necesario plasmar mediante diversas actuaciones la situación que presentan los ámbitos de la documentación y la investigación aplicados, en este caso, a la profesión y el trabajo cinematográficos, estructurados en un amplio abanico de realizaciones (gestión de la información, tratamiento documental, metadatos, web semántica, digitalización, edición electrónica fuera de línea y en línea, posicionamiento en Internet, tipologías de fondos fílmicos en red, interconexión vía web de filmotecas y otras instituciones especializadas, formación semipresencial y virtual, patrimonio cinematográfico...), así como la evolución que dichos ámbitos están experimentando constantemente en función del propio desarrollo de la sociedad de la información y de las tecnologías digitales (López Yepes et álii, 2006).

Por otra parte, las temáticas mencionadas deben contemplarse, según nuestro criterio, con una visión presente y de futuro que incluya los puntos de vista alternativos que proporcionan las experiencias, aplicaciones y desarrollos en y desde diversos ámbitos profesionales y académicos: la universidad y otras instituciones de estudio e investigación, presenciales, semipresenciales y virtuales; las filmotecas 
y archivos fílmicos; los medios de comunicación cinematográficos (prensa escrita, programas monográficos de cine en radio y televisión, sitios en Internet, etcétera); las asociaciones, fundaciones y comisiones cinematográficas y otras instituciones especializadas e implicadas en el trabajo cinematográfico.

La propuesta que aquí y ahora se plantea, desgajada de las relacionadas más arriba, incide y se centra especialmente en la colocación (publicación) de fondos fílmicos en red y en la interconexión de los mismos y de las propias instituciones que los albergan (fundamentalmente si disponen de los derechos correspondientes para su difusión).

El tema ya fue esbozado y sugerido por el autor de estas páginas, pero de forma bastante más sintetizada, el día 26 de mayo de 2006 en Barcelona, con motivo de la celebración de las IV Jornades de Documentació Audiovisual, en el marco de las $\mathrm{X}$ Jornades Catalanes de Informació i Documentació que tuvieron lugar entre los días 24 y 26 de mayo de 2006.

\section{Filmotecas y archivos fílmicos en Internet: situación actual}

En la actualidad son 15 las filmotecas de comunidades autónomas que están prestando sus servicios, y 13 de ellas disponen en estos momentos de página web. Tan solo las filmotecas de Asturias y Castilla y León (en Salamanca) no están presentes todavía en la red de redes. Se relacionan a continuación dichas instituciones, indicándose el año de creación y su dirección web (Cuadra, 2006a), en junio de 2006:

Filmoteca Vasca / Euskadiko Filmategia (1978)

http://www.paisvasco.com/filmoteca/

Filmoteca de Zaragoza (1981)

http://www.zaragozafilmo.tk/

Filmoteca Española (1982)

http://www.mcu.es/jsp/plantilla_wai.jsp?id=11\&area=cine

Filmoteca de la Generalitat de Catalunya (1982)

http://cultura.gencat.net/filmo/

Filmoteca Canaria (1984)

http://www.culturadecanarias.com/filmotecac/

Filmoteca de Murcia (1984-1996) (Filmoteca Regional Francisco Rabal)

http://www.filmotecamurcia.com/

Filmoteca de la Generalitat Valenciana (1985)

http://www.ivac-lafilmoteca.es/

Filmoteca de Andalucía (1989)

http://www.filmotecadeandalucia.com/

Scire. 14 : 2 (jul.-dic. 2008) 41-64. ISSN 1135-3716. 
Centro Galego de las Artes da Imaxe (CGAI) (1991)

http://www.cgai.org/

Filmoteca de Castilla y León (1991)

Filmoteca de Asturias (1996)

Arxiu del So i la Imatge (ASIM) (Baleares) (1998)

http://www.conselldemallorca.net/biblioteques/fonssoim.htm

Filmoteca de Cantabria (2001)

http://www.palaciofestivales.com/cine.html

Filmoteca de Albacete (2001). (A partir de 2007 está prevista su reconversión en Filmoteca de Castilla - La Mancha, dependiendo de la Junta de Castilla La Mancha).

http://www2.amialbacete.com/filmoteca

Filmoteca de Extremadura (2003)

http://www.filmotecaextremadura.com

Se han hecho estudios recientemente sobre el aspecto formal de las páginas web de filmotecas ("análisis externo") y sobre si los contenidos son adecuados, pertinentes y suficientes en el ámbito de estas instituciones (“análisis interno") (Cuadra, 2006a).

Dichos análisis se concretan en la comprobación de una serie de parámetros e indicadores y en la accesibilidad. Dichos parámetros e indicadores se concretan en los siguientes puntos: 1) contenido: calidad y volumen de la información; 2) autoría: responsabilidad y solvencia; 3) legibilidad y ergonomía; 4) navegación y recuperación de la información; 5) interactividad; 6) velocidad de descarga; 7) enlaces: luminosidad, calidad, actualización y evaluación.

Como conclusión puede afirmarse que las filmotecas españolas estimulan el acceso al patrimonio cinematográfico y que la mayoría de ellas promueve la difusión del patrimonio, anima a su conservación y, al mismo tiempo, estimula su visionado. El apartado que más se trata en las sedes web de las filmotecas es el que se refiere a las proyecciones, la manera más válida de difundir el patrimonio. En estos momentos se puede acceder a las bases de datos de sus películas, a los catálogos de sus bibliotecas y a las programaciones de sus ciclos, entre otros servicios (Torrado, 2006). No obstante, se echa en falta una mayor diversidad de contenidos accesibles en línea, que mejorarían considerablemente la riqueza de los sitios. Podrían mencionarse los siguientes puntos (Gómez, 2006):

- Inexistencia vídeos y documentos sonoros: por ejemplo, podría haber vídeos con fragmentos de películas restauradas, muestras de trabajos de restauración de obras, archivos sonoros con fragmentos de bandas sonoras y otros materiales de interés para investigadores, profesionales o cinéfilos.

Scire. 14 : 2 (jul.-dic. 2008) 41-64. ISSN 1135-3716. 
- Colaboración entre instituciones y proyectos en común: entre otras actuaciones, la propuesta de una base de datos común de fondos de filmotecas.

- Espacio de comunicación entre los usuarios: una especie de foro libre con el que aumentar la interactividad de la página y permitir a los usuarios intercambiar opiniones y experiencias o compartir intereses y aficiones.

- Posibilidad de personalización de la página para cada usuario o establecimiento de un perfil personalizado de navegación: acceso rápido a los contenidos preferidos o más visitados, servicio de alerta sobre la proyección de determinados ciclos (por directores, géneros, estilos...), etcétera.

- Difusión selectiva de la información: configuración de un servicio de envío de boletines de información en función de las distintas categorías e intereses de los usuarios: nuevas publicaciones, próxima exhibición de películas, futuros convenios o proyectos cooperativos, etcétera.

\section{El ámbito universitario: CineDocNet, portal de documentación cinematográfica}

CineDocNet fue creado en 2002 como consecuencia o resultado de un proyecto de innovación educativa financiado por el Vicerrectorado de Investigación de la Universidad Complutense. Se trata de un portal diseñado y mantenido en el ámbito del Servicio de Documentación Multimedia - Multidoc del Departamento de Biblioteconomía y Documentación de la Facultad de Ciencias de la Información de Madrid (López Yepes y Pérez Agüera, 2002).

Tras una primera fase del portal diseñado con software de código abierto PHPNuke y PHPPostNuke, y una segunda fase estructurada con MAMBO, se ha dado paso a una tercera época con JOOMLA, y la siguiente estructura sistematizada de contenidos:

Mediateca (hemeroteca, fototeca, fonoteca, videoteca...)

Noticias - aportaciones

Bibliografía - webgrafía - videografía

Foros

Lista distribución (CineDoc)

Chat

Enlaces

Buscador general

Guía del usuario - Regístrate - Sitemap

Algunos ejemplos contenidos y accesibles en línea a través del portal CineDocNet han sido recogidos en la videoteca de la Mediateca (López Yepes, 2003), incluyendo varias investigaciones de curso en el marco de prácticas de la asignatura Documentación de 5..$^{\circ}$ curso, de la sección Documentación Audiovisual del Departamento de Documentación. Se incluyen archivos fílmicos en diversos formatos (avi, mpg, wmv, mov) y duraciones. Dichos documentos digitales tienen procedencia muy diversa: revista Cuadernos de Documentación Multimedia (ac- 
cesible desde el portal), pases de prensa de películas con sus correspondientes tráilers, preestrenos, festivales, documentales y entrevistas personalizadas realizadas por el autor de este trabajo, investigaciones de curso realizadas en el marco práctico de la asignatura Documentación, que se imparte en las licenciaturas de Ciencias de la Información (Periodismo, Comunicación Audiovisual y Publicidad y Relaciones Públicas) y en la licenciatura de Documentación.

Se relacionan a continuación algunos de estos documentos y estudios documentales en materia cinematográfica, con temáticas muy diversas y la mayor parte de ellos con contenidos multimedia (vídeo digital), referenciados en el portal y producidos desde 1996: La documentación del guión cinematográfico; Revista Cinerama: proceso documental; Escuelas y festivales de cine en Internet; Aplicación de bases de datos en festivales de cine; La documentación en el proceso de iluminación de una película; La documentación de los críticos cinematográficos; Monográficos de cine en televisión: TVE (Días de cine) - Telemadrid (El Megahit) Canal Plus (MagaCine); Qué grande es el cine; Filmoteca Española: historia y proceso de preparación de ciclos cinematográficos: ciclo Alfred Hitchcock; La restauración en las filmotecas; La documentación en las películas de época: entrevistas a Juan Amorós y Carlos Saura a propósito de El Dorado; La documentación en la dirección artística: entrevista a Félix Murcia; Art Futura: historia, actividades, programación; Empresas infográficas españolas e imagen digital en el cine español: Brainstorm Multimedia / Computer Arts \& Development (CAD); La documentación en el guión cinematográfico; La Filmoteca Española; ¿Cómo se documenta el actor?; La documentación de los programas de cine en televisión; El servicio de documentación multimedia de RTVE Cartelera; El ordenador en el montaje cinematográfico; El proceso de documentación en los cines Alphaville; Cine Informe; Aportaciones del cine actual a la banda sonora; La documentación del escritor: Gonzalo Suárez; La filmoteca de Castilla y León; "Movie Magic". El sistema informático en la organización de un rodaje; La recuperación y restauración del patrimonio cinematográfico español; Guía de fuentes sobre el cine español; Distribución y marketing cinematográfico; La censura en el cine español; El cine y la guerra civil española; Cine deportivo; El centro de documentación cinematográfica; Documentación en "Luces de bohemia": el rodaje, 1985 - decorados - ambientación; Artimaña Producciones; Bases de datos cinematográficas; Fotofilm España; Network; SGAE; Videonet; Enciclopedia del Cine Español: contenidos; Semana Internacional de Cine de Valladolid (SEMINCI); Restauración cinematográfica (Filmoteca Española); Efectos digitales en cinematografía $(C A D) ;$ Exposición "Directores artísticos"; Futuroscope; La documentación en la Universidad Complutense; Festival de Cine Independiente de Elche; Festival de Cine Iberoamericano de Huelva; Festival de Cine Iberoamericano de Villaverde - Madrid; Filmoteca Española: "Salvar el cine" (documental); Filmoteca

Scire. $14: 2$ (jul.-dic. 2008) 41-64. ISSN 1135-3716. 
Española - conservación: Ramón Rubio; Filmoteca Española - restauración: Alfonso del Amo; Iluminación cinematográfica; Edición no lineal; Producción-distribución cinematográficas; Cine en televisión; Cineinforme - Exportfilm; Casa de América; Agencia EFE; Kodak: postproducción; Telson: postproducción; Cine digital: Peter Greenaway...

Obviamente, resulta imprescindible la difusión informativa de todos los contenidos del portal, y muy especialmente los del ámbito de la Mediateca del mismo, en la que se integra la videoteca digital y, en consecuencia, numerosos y variados documentos fílmicos procedentes de pases de prensa de películas, preestrenos, entrevistas con profesionales del cine, grabaciones en festivales de cine, investigaciones de curso y estudios documentales multimedia como los que se acaban de mencionar, etcétera. Algunos modos de difusión que ya se están aplicando o cuya instrumentalización se efectuará próximamente se especifican a continuación:

- De modo general, establecer una buena estrategia de difusión informativa de la existencia y funcionamiento del portal, teniendo en todo momento claro a qué tipología de usuarios está dirigido (en función de qué usuarios reales y potenciales se ha generado el portal).

- Difusión informativa a través del posicionamiento en motores de búsqueda, gratuitamente o mediante pago, en pro de la visibilidad más óptima, y con una buena definición en cuanto a metadatos que facilite la indexación temática por parte de los buscadores.

- A través de ámbitos profesionales relacionados, como por ejemplo jornadas, congresos, festivales...

- Conseguir patrocinio de eventos vinculados con la materia; intercambio de banners con otros sitios de temática relacionada; anunciarse en buscadores (por ejemplo, Google AdWords); intercambio de enlaces con organismos afines (filmoteca y otros archivos fílmicos, agencias de publicidad, asociaciones profesionales, etcétera)...

- Usar las listas de distribución especializadas o relacionadas; brindar la posibilidad de formularios en línea para incorporar nuevos vínculos al sitio y nuevas direcciones para su difusión.

- Actividades cooperativas donde se compartan recursos informativos, resultados y publicidad conjunta, lo que supondría la interconexión vía web de la producción informativa (vinculación, por ejemplo, y si fuera posible, con el depósito legal).

- Utilizar asimismo uno de los también mejores medios de difusión informativa: el boca a boca.

- A raíz de la existencia del portal, y de la oferta de colaboración con otras instituciones e instancias especializadas, propuesta de un centro de documentación común que coordine los de cada una de las empresas. Esto viene a significar en realidad la propuesta, ya anunciada en otras ocasiones y publicaciones, de una verdadera "comunidad virtual de usuarios de documentación cinematográfica".

- Planteamiento de realización de campañas publicitarias sobre la existencia del portal dentro del sector profesional, por ejemplo en el ámbito de festivales, certámenes de cine u otros campos temáticos en estrecha relación con el cine y derivados...

Scire. 14 : 2 (jul.-dic. 2008) 41-64. ISSN 1135-3716. 
- Difusión informativa vía guías de lectura específicas de cine, en publicaciones de cine-clubs, cine-fórums, etcétera.

- Establecer contactos con todo tipo de ámbitos de formación tanto nacionales como internacionales (iberoamericanos, por ejemplo), con el fin de intercambiar vínculos de portales, etcétera.

- Asimismo, contacto con empresas de producción, distribución y exhibición cinematográficas y otras relacionadas con el mundo del cine.

- Proyectos de colaboración en distintos niveles con entidades diversas, estableciendo sinergias enfocadas a resaltar su utilidad en todo tipo de ámbitos relacionados con el cine en todos sus aspectos.

En fin, el portal está integrado, inicialmente, en una red temática iberoamericana de documentación informativa - InfoDocNet—, que fue presentada en las jornadas Posicionamiento.es. El posicionamiento en Internet de instituciones culturales, científicas y educativas, celebradas en la Universidad de Alicante entre los días 5 y 7 de mayo de 2004 (López Yepes, 2004).

\section{Filmotecas y archivos fílmicos en línea: posicionamiento en Internet}

En el mundo digital los metadatos se han incorporado para facilitar la gestión de los archivos y la navegación en red. La creación e implementación de metadatos es un proceso intensivo que requiere una importante inversión de tiempo, recursos humanos y económicos, por lo que es necesario hacer un balance de costos y beneficios teniendo en cuenta las necesidades de los usuarios y de los administradores de la colección actuales y futuros.

Una buena descripción del contenido de los documentos cinematográficos, basada en la aplicación de los metadatos más adecuados y en el buen uso de lenguajes de descripción multimedia, contribuye a un buen posicionamiento (visibilidad) en Internet. Se facilita, asimismo, por parte de los buscadores de la Red una indexación temática lo más pertinente posible (López Yepes, Sánchez Jiménez y Pérez Agüera, 2003). Conviene recordar en este punto que en el ámbito de la Web se trabaja con sistemas como el Synchronized Multimedia Integration Language (SMIL) para la gestión de documentación multimedia (Gil Urdiciaín, Pérez Agüera y Sánchez Jiménez, 2004), y cada vez con más profusión en entornos distribuidos de trabajo en colaboración, cuyo fin último es obtener la configuración de un sistema multiagente de recuperación de información (López Yepes, Sánchez Jiménez y Pérez Agüera, 2005b).

Se distinguen tres categorías de metadatos, atendiendo a las funciones que desempeñan y a la información que se desea ofrecer: metadatos descriptivos, cuyo objetivo es la descripción e identificación de los recursos de información, ya sea en un sistema local o en una aplicación web para ayudar a los usuarios en la localización de recursos; metadatos estructurales, que facilitan la navegación y la 
presentación de los recursos electrónicos proporcionando información sobre la estructura interna de los mismos: página, sección, capítulo, numeración, índices, tablas de contenidos; y metadatos administrativos, que posibilitan la gestión y el procesamiento de las colecciones digitales, e incluyen datos técnicos sobre la creación y el control de calidad, la gestión de derechos, el control de acceso y utilización y las condiciones de preservación.

Por otra parte, el Moving Pictures Experts Group (MPEG) ha sido responsable del desarrollo de estándares para la codificación de información audiovisual. La conexión de MPEG con el mundo de los metadatos da como resultado el estándar MPEG-7, denominado interfaz de descripción de contenidos multimedia. Es importante destacar el desarrollo del nuevo estándar MPEG-21 (el más reciente) para resolver las limitaciones de los anteriores. Este esquema está orientado a la infraestructura necesaria para la distribución y el uso de contenidos digitales, y abarca cuestiones relativas a la producción, descripción, gestión, protección y acceso.

En resumen,

Los documentos digitales tienen unas características físicas que los hacen mucho más efímeros, volátiles y vulnerables que los documentos tradicionales. Junto a esto, la vertiginosa evolución tecnológica a la que asistimos ha contribuido a acelerar la caducidad de equipos, soportes y formatos de ficheros, factores todos ellos que pueden afectar de forma considerable a la integridad de los archivos y a la protección del contenido. [...] Una parte de la memoria de la Humanidad puede quedar inaccesible, invisible, inexistente... [...] Es urgente por lo tanto desarrollar estrategias técnicas, de organización y de gestión, sobre todo en lo que se refiere al seguimiento de normas y directrices internacionales que garanticen la conservación y acceso a largo plazo de los recursos digitales. Sin lugar a dudas, el desarrollo de un marco general de metadatos de preservación va a contribuir a la interoperatibilidad futura de los archivos digitales además de compartir recursos y facilitar el intercambio de metadatos" (Salvador y Ruiz, 2005-2006).

\section{Digitalización de fondos fílmicos e interconexión de filmotecas: una propuesta necesaria}

Como preámbulo al planteamiento de digitalización del patrimonio cultural (en este caso cinematográfico) consideramos de enorme interés mencionar la Carta de la UNESCO de 15 de octubre de 2003 y reproducir parte de sus contenidos (Nistal, 2006):

Preámbulo

La Conferencia General,

Considerando que la desaparición de cualquier forma de patrimonio empobrece el acervo de todas las naciones,

Recordando que la Constitución de la UNESCO establece que la Organización debe ayudar a la conservación, al progreso y a la difusión del saber, velando por la conservación y la protección del patrimonio universal de libros, obras de arte y monumentos

Scire. 14 : 2 (jul.-dic. 2008) 41-64. ISSN 1135-3716. 
de interés histórico o científico, que su Programa Información para Todos ofrece una plataforma para el debate y la acción sobre políticas de información y sobre la salvaguardia de los conocimientos conservados en forma documental, y que su programa "Memoria del Mundo" tiene por objeto garantizar la preservación del patrimonio documental del mundo y un acceso universal al mismo,

Reconociendo que esos recursos de información y expresión creativa se elaboran, distribuyen, utilizan y conservan cada vez más en forma electrónica, y que ello da lugar a un nuevo tipo de legado: el patrimonio digital,

Consciente de que el acceso a dicho patrimonio brindará mayores oportunidades de creación, comunicación e intercambio de conocimientos entre todos los pueblos,

Entendiendo que este patrimonio digital se encuentra en peligro de desaparición, y que su preservación en beneficio de las generaciones actuales y futuras es una preocupación urgente en el mundo entero,

la Carta sobre la preservación del patrimonio digital de la Unesco insta a las organizaciones internacionales, gubernamentales y no gubernamentales, y el sector privado, a adoptar todas las medidas necesarias para aplicar esta Carta.

\section{Artículo 2. Acceso al patrimonio digital}

El objetivo de la conservación del patrimonio digital es que este sea accesible para el público. Por consiguiente, el acceso a los elementos del patrimonio digital, especialmente los de dominio público, no debería estar sujeto a requisitos poco razonables. Al mismo tiempo, debería garantizarse la protección de la información delicada o de carácter privado contra cualquier forma de intrusión. Los Estados Miembros tal vez deseen trabajar en colaboración con las organizaciones e instituciones pertinentes para propiciar un contexto jurídico y práctico que maximice la accesibilidad del patrimonio digital. Convendría reafirmar y promover un justo equilibrio entre los derechos legítimos de los creadores y otros derechohabientes y el interés del público por tener acceso a los elementos del patrimonio digital, de conformidad con las normas y los acuerdos internacionales.

\section{Artículo 3. El peligro de pérdida}

El patrimonio digital del mundo corre el peligro de perderse para la posteridad. Contribuyen a ello, entre otros factores, la rápida obsolescencia de los equipos y programas informáticos que le dan vida, las incertidumbres existentes en torno a los recursos, la responsabilidad y los métodos para su mantenimiento y conservación y la falta de legislación que ampare estos procesos. Los cambios en las conductas han ido a la zaga del progreso tecnológico. La evolución de la tecnología digital ha sido tan rápida y onerosa que los gobiernos e instituciones no han podido elaborar estrategias de conservación oportunas y bien fundamentadas. No se ha comprendido en toda su magnitud la amenaza que pesa sobre el potencial económico, social, intelectual y cultural que encierra el patrimonio, sobre el cual se edifica el porvenir.

Artículo 4. Necesidad de pasar a la acción

A menos que se haga frente a los peligros actuales, el patrimonio digital desaparecerá rápida e ineluctablemente. El hecho de estimular la adopción de medidas jurídicas,

Scire. 14 : 2 (jul.-dic. 2008) 41-64. ISSN 1135-3716. 
económicas y técnicas para salvaguardar ese patrimonio redundará en beneficio de los propios Estados Miembros. Urge emprender actividades de divulgación y promoción, alertar a los responsables de formular políticas y sensibilizar al gran público tanto sobre el potencial de los productos digitales como sobre los problemas prácticos que plantea su preservación.

\section{Artículo 6. Elaborar estrategias y políticas}

Es preciso elaborar estrategias y políticas encaminadas a preservar el patrimonio digital, que tengan en cuenta el grado de urgencia, las circunstancias locales, los medios disponibles y las previsiones de futuro. La colaboración de los titulares de derechos de autor y derechos conexos y otras partes interesadas a la hora de definir formatos y compatibilidades comunes, así como el aprovechamiento compartido de recursos, pueden facilitar esa labor.

Y hemos de destacar, en palabras de la autora mencionada al inicio del epígrafe, que "Una buena colección digital debe mantenerse en el tiempo. En particular las colecciones digitales deben disponer de un plan que les permita durar y mantenerse en el tiempo, más allá del proyecto que las originó. Un objeto digital es persistente. Esto es, que será accesible a lo largo del tiempo a pesar de que haya cambios tecnológicos. Se digitaliza en un formato que soporta el uso actual y futuro o que soporta el proceso de crear copias que faciliten el uso. Un buen objeto se puede intercambiar entre plataformas, es ampliamente accesible y se digitaliza de acuerdo a las mejores prácticas disponibles".

Asimismo, consideramos sumamente ilustrativas las conclusiones emanadas de las jornadas celebradas en Madrid entre los días 14 y 16 de marzo de 2006 sobre La preservación del patrimonio digital: conceptos básicos y principales iniciativas:

- Se considera de urgente necesidad que el Ministerio de Cultura y los departamentos de las Comunidades Autónomas responsables en materia de Cultura inicien acciones administrativas y legales dirigidas a la protección del patrimonio digital como parte integrante del Patrimonio Cultural, para su difusión, investigación y transmisión a las generaciones futuras.

- Resulta prioritario establecer una estrategia nacional de preservación digital que implique a todas las administraciones, instituciones, entidades y agentes relacionados con el patrimonio cultural y la producción de contenidos.

- Es imprescindible abordar con un planteamiento cooperativo la planificación, gestión y ejecución de las acciones de preservación digital, de forma que sea posible compartir las responsabilidades y distribuir las tareas entre todos los organismos implicados.

- Se recomienda la creación, bajo la iniciativa y coordinación del Ministerio de Cultura, de una red de conocimiento sobre preservación digital para que los profesionales de las bibliotecas y de otras instituciones de la memoria, como archivos y museos, identifiquen esta área como sector propio de su actividad profesional. En los contenidos de dicha red se dará especial relevancia a los aspectos de normalización y a las buenas prácticas.

Scire. 14 : 2 (jul.-dic. 2008) 41-64. ISSN 1135-3716. 
- Se propone la elaboración de un modelo conceptual y la creación de una plataforma tecnológica que permitan la puesta en marcha, desarrollo y difusión de experiencias piloto en los distintos ámbitos de actividad de la preservación digital.

Un claro e interesante ejemplo de digitalización de fondos fílmicos y de su acceso a través de Internet es el de British Pathé (Cuadra, 2006b), que supone la posibilidad de "mostrar al mundo los materiales que custodian, sirviendo a investigadores de todo el planeta, así como ampliar el público y las instituciones que podrían acceder a la compra de este material a través de Internet. Este archivo fílmico es uno de los más antiguos y es el primer archivo de material informativo que ha digitalizado sus fondos. Gracias a las aportaciones del Fondo Lottery Funded New Opportunities Fund fue posible digitalizar el fondo y crear esta página web. (Esta fundación recibe la mitad de los beneficios que genera la lotería nacional en el Reino Unido, y dedica estos fondos a obras benéficas, becas, programas culturales, etc.)". "Los materiales de British Pathé se digitalizaron con el espíritu de que cualquiera pudiera acceder a los archivos, siempre con fines didácticos. El uso profesional del material exige el pago de derechos, pero cualquier usuario de Internet puede acceder a una 'pre-visualización' o preview, con poca calidad y con marca de agua, pero se puede acceder a las imágenes mediante búsquedas sencillas y avanzadas". "En la página principal de British Pathé se ofrece algo de información acerca de la historia del archivo, de la institución que patrocina la digitalización del archivo. El material se ofrece gratuitamente para las escuelas de Inglaterra".

En otro ámbito, este fundamentalmente informativo, se dispone de un directorio de fuentes y de recursos fílmicos en Internet (López Yepes, 2001) y de un proyecto de investigación sobre el cine en la infopista (López Yepes, 2002). Elaborados y codirigidos en su día en el ámbito del Servicio de Documentación Multimedia de la Universidad Complutense por el autor de estas páginas, suponen dos obras de consulta o referencia que conviene mantener permanentemente actualizadas. Y, de otra parte, es necesario conocer la evolución que representan las iniciativas open access que afectan a la comunicación y la documentación cinematográficas (Arencibia, Santillán y Subirats, 2005).

Asimismo, debe mencionarse necesariamente el Proyecto Piloto de Digitalización que a efectos oficiales se ha llevado a cabo en el ámbito de Filmoteca Española y en el marco de Patrimonio.es (Nistal, 2006). Se trata de una actuación experimental basada en la digitalización de una selección de películas anteriores a 1953 y materiales asociados, y la producción de una serie de másters digitales y derivados de materiales fílmicos y no fílmicos en estrecha relación con la historia del cine español. Las prioridades de Patrimonio.es se sustentan en la digitalización, la difusión y la colaboración con instituciones, y más concretamente en la convocatoria de proyectos como el que se acaba de mencionar. Añadimos una serie de conclusiones en relación con la digitalización del patrimonio cultural español:

Scire. $14: 2$ (jul.-dic. 2008) 41-64. ISSN 1135-3716. 
— La información es ya mayoritariamente digital.

- Cualquier previsión, aún la más conservadora, indica que seguirá creciendo a ritmos mayores que los actuales, especialmente en cuanto a contenidos en lenguas españolas.

- Los nuevos modos de transmisión de información obligan a nuevos modos de gestión.

- El problema no afecta solo a bibliotecas y archivos, filmotecas, fonotecas, fototecas y videotecas.

- La colaboración entre sectores y organismos es fundamental.

- El uso y fomento de estándares es vital en este proceso.

- Hay que tener en cuenta los "nuevos" problemas legales que aparecen en este ámbito.

Con anterioridad a este proyecto piloto de la Filmoteca Española, la Filmoteca de Catalunya digitalizó y publicó, en tres CD-ROM correspondientes a los años 2001 (Filmoteca de Catalunya, 2001), 2004 (Filmoteca de Catalunya, 2004) y 2005 (Filmoteca de Catalunya, 2005), fondos de nitratos de films de ficción y de no ficción. Son en estos momentos 700 las horas que dicha institución ha digitalizado en relación con el cine catalán (cine primitivo y cine documental): se encuentran accesibles únicamente vía intranet, en su sala de consulta. Asimismo, esta Filmoteca tiene en proyecto, juntamente con las Filmotecas Española y el IVAC valenciano, la publicación de un catálogo del cine español hasta 1950.

Por otra parte, con motivo de la celebración del último Congreso de la Federación Internacional de Archivos de Films (FIAF), en abril de 2006, en Brasil, se informó acerca de la digitalización que se está llevando a cabo en filmotecas y archivos fílmicos alemanes e italianos, fundamentalmente.

En fin, ciñéndonos al ámbito del patrimonio cinematográfico español, se avanzan una serie de propuestas de actuaciones que inevitablemente pueden mejorar la situación actual.

Una de esas propuestas de actuación se enfoca hacia el planteamiento, como apuntamos anteriormente, de que filmotecas y archivos fílmicos (y sus fondos, o parte de los mismos) estén interconectados vía web y puedan ser recuperados por investigadores, estudiosos, etcétera. Y uno de los fundamentos se localiza, por ejemplo, en el 17 de mayo de 2005, en el marco de la 58. ${ }^{\text {a }}$ edición del Festival de Cine de Cannes. Se celebraba entonces por tercer año consecutivo el Día de Europa, bajo el título "Películas Europeas y la Sociedad de la Información", y como resultado de ello se establecía la "Declaración de Cannes 2005". De entre su amplio articulado y conclusiones destacamos, porque afectan especialmente a la documentación y la investigación cinematográficas, y señalan la influencia de Internet en todo el ámbito cinematográfico, los siguientes aspectos: 1) facilitar el desarrollo de una industria cinematográfica on-line europea respondiendo a los desafíos planteados (liberación de derechos, ventanas de explotación); 2) prever mecanismos financie- 
ros (en MEDIA 2007) para el lanzamiento de servicios de distribución on-line europeos, y para la digitalización de las obras; 3) examinar cómo los servicios on-line pueden contribuir a la promoción de la producción de contenidos audiovisuales originales; 4) estimular el acceso al patrimonio cinematográfico.

Añadido a esto, conviene también ahora mencionar las propuestas que sobre el establecimiento de una biblioteca digital europea e incluso de una biblioteca digital universal están proliferando, actuaciones emanadas y emprendidas por la Unesco y otras instituciones en esta línea; muy especialmente en fechas recientes, y cada vez con más motivo en función de los desarrollos de la sociedad de la información, de la sociedad multimedia. Véanse al respecto los avances en relación con el mayor ancho de banda presentados en Cebit 2006 y otros foros y ferias especializados. Podría incluso, a propósito de esta propuesta de interconexión vía web de fondos fílmicos, ampliarse o proponerse el término filmoteca digital universal, o bien englobarse en el de biblioteca, aunque con los matices correspondientes.

Algunas otras pautas de trabajo se proponen y relacionan a continuación:

- Solicitar apoyos en el marco de instituciones de investigación, ya sea en el ámbito nacional como en el internacional (como Patrimonio.es o eContent Programme).

- Esos acuerdos y proyectos tendrían que extenderse a instituciones no exclusivamente cinematográficas, aunque sí relacionadas (tanto públicas como privadas).

- Para proceder al conocimiento global y la difusión de cuál es la situación del patrimonio fílmico español y de la labor que realizan las filmotecas, tendrían que relacionarse con grandes redes académicas. Un ejemplo de ello es la red latinoamericana que interconecta las redes de investigación nacionales de América Latina a través de su sitio web RedCLARA, sobre la que se facilita más información en un epígrafe posterior.

- Sería recomendable que las filmotecas españolas tuvieran una base de datos común accesible desde Internet. De esta forma un usuario podría localizar en España una película o unas imágenes sin moverse de casa. Además se debería incluir un apartado desde el que acceder al visionado de algunas secuencias claves de la historia del cine español.

- Por otro lado, la financiación es un punto fundamental. Por ello sería interesante contar con el apoyo de instituciones privadas que apoyaran la difusión del patrimonio cinematográfico. También habría que aumentar las acciones encaminadas a localizar fondos cinematográficos españoles en el extranjero y a realizar algunas copias de dichos fondos (creo que en este momento se está colaborando con el archivo argentino Difilm). La financiación también debería estar enfocada a mejorar las instalaciones y a digitalizar los fondos. Es una pena que un usuario tenga que esperar alrededor de dos meses para visionar material en la Filmoteca Española.

- Podría estudiarse la creación de diferentes productos de tipo educativo, cultural o científico, así como la elaboración de programas de investigación, becas, premios, etcétera.

- La situación de los archivos fílmicos ante el desarrollo tecnológico tiene que pasar obligatoriamente por un plan global de digitalización.

Scire. 14 : 2 (jul.-dic. 2008) 41-64. ISSN 1135-3716. 
- Se debería realizar un análisis preliminar de la situación para proponer un entorno digital que tuviera en cuenta documentación, duplicados para consulta, restauraciones de sonido e imagen, depósito de nuevos materiales, generación de contenidos...

- Posteriormente podría analizarse el modelo de datos necesario para compatibilizar las bases de datos documentales de las filmotecas españolas con los patrones de intercambio que se utilizan en el resto de filmotecas españolas y europeas para posibilitar el intercambio de fondos.

- Se podría contemplar la creación de un portal en la Red que pudiera ofrecer copias de los archivos en calidades baja y media, suficientes para la consulta con fines científicos, pedagógicos, culturales o de ocio.

- Pero todo esto exige un compromiso de determinadas organizaciones, con frecuencia las encargadas de la preservación de la memoria colectiva, tales como archivos, bibliotecas y museos (tanto del ámbito público como del privado). El uso de tecnologías y herramientas de digitalización obliga a estas instituciones culturales a adquirir nuevas competencias y prácticas; son actividades costosas a largo plazo y técnicamente exigentes. Por ello se debe procurar la implicación de sectores económicos e institucionales que puedan financiar y colaborar en el desarrollo de estos proyectos.

- Todo ello debe dotarse de una normativa clara y conviene dar soluciones a la gestión de los derechos si se quiere determinar el valor económico de los contenidos de forma sostenible.

- Finalmente, como ejemplo ilustrativo de actuaciones que tienen lugar en otros países, en este caso en Latinoamérica, en México se destina un peso de cada entrada de cine a la construcción de una filmoteca nacional que permitirá concentrar en un solo lugar y bajo las condiciones apropiadas el amplio y rico acervo audiovisual mexicano. Desde hace unos años se organiza en Ciudad de México el Seminario Internacional de Archivos Sonoros y Audiovisuales, que ha servido para ir creando conciencia de la importancia de la conservación del patrimonio sonoro y audiovisual de ese país. Es el comienzo para que esta concienciación acerca de la conservación del patrimonio fílmico mexicano llegue al ámbito del Gobierno federal y los Gobiernos locales con objeto de que se desarrollen políticas públicas y se destine una partida presupuestaria a estos fines.

\section{Proyecto Filmoteca de Andalucía - Servicio de Documentación Multimedia (Departamento de Biblioteconomía y Documentación de la Universidad Complutense)}

Con el espíritu al que se ha aludido (digitalización de fondos fílmicos), y con el ánimo de continuar con otras aportaciones en la misma o parecida línea de actuación, se acaba de establecer (en abril de 2006) un convenio de colaboración entre el Servicio de Documentación Multimedia del Departamento de Biblioteconomía y Documentación de la UCM y la Filmoteca de Andalucía, dependiente de la Consejería de Cultura. Canalizado a través de la OTRI (Oficina de Transferencia de Resultados de Investigación) de la Universidad Complutense, dicho proyecto supo-

Scire. 14 : 2 (jul.-dic. 2008) 41-64. ISSN 1135-3716. 
ne el establecimiento en Internet de un portal con los fondos fílmicos del cineasta español José Val del Omar (artista polifacético-multimedia), depositados en la mencionada Filmoteca (al igual que los del director de fotografía Teo Escamilla), y toda una labor de difusión de información cinematográfica distribuida vía web para su recuperación por parte de profesionales muy diversos y usuarios en general interesados en el tema.

Dicho proyecto se estructura en las siguientes líneas de actuación y fases de trabajo:

- Adquisición, ya realizada, por parte de la Filmoteca de Andalucía del dominio http://www.valdelomar.org / http://www.valdelomar.com, que albergará el fondo documental-filmográfico en línea (en realidad ya se ha iniciado la creación de contenidos). En su momento se establecerá un enlace con el servidor del Servicio de Documentación Multimedia - Multidoc, http://multidoc.rediris.es, con objeto de conseguir una difusión todavía mayor y como materialización de la presencia de ambas instituciones en el proyecto de investigación.

- Inventario de materiales y documentos.

- Diseño y creación del sitio en Internet: labores de escaneado, catalogación y digitalización de documentos.

- Otras actividades que se llevarán a cabo de forma simultánea y posterior a la creación del sitio $\mathrm{VdO}$ (Val del Omar) en Internet. El sitio creado al efecto funcionará permanentemente como instrumento de difusión informativa de dichos desarrollos:

- Experiencia VdO: un espacio compartido con artistas e intelectuales, germen de la influencia e inspiración de Val del Omar.

- Biografía e historia: infancia y formación (París, Granada). Juventud (Madrid, Residencia de Estudiantes, Misiones Pedagógicas, guerra). Posguerra (Valencia, Madrid). Años sesenta y setenta. Últimos años. Contexto histórico (historia de aquellos años).

- Su estudio: el espacio, condiciones de vida. Biblioteca del estudio. Herramientas (equipos, prototipos, otros).

- Creación y obra: Textos. Cine (filmografía, proyectos y guiones, inventario de materiales conservados). Acariño galaico (historia de su restauración). Audio. Plástica. Fotografía. Otras experiencias.

- Pensamiento.

- Invención técnica.

- Prensa (vaciados de época).

- Escenarios VdO: la Alhambra, Museo Escultura de Valladolid, catedral de Santiago.

- Actualidad. Noticias. Centenario.

- Glosario (terminología VdO).

- Foro dirigido.

- Premio Val del Omar.

- Bibliografía - webgrafía.

- Etcétera.

Scire. 14 : 2 (jul.-dic. 2008) 41-64. ISSN 1135-3716. 


\section{Monográfico de Cuadernos de Documentación Multimedia}

Con idéntico ánimo de concreción y difusión de la propuesta de digitalización e interconexión vía web de las filmotecas se orienta la propuesta de elaboración en colaboración de un número especial (16, 2005-2006) de Cuadernos de Documentación Multimedia, revista nacida en 1993 en el seno del Servicio de Documentación Multimedia - Multidoc del Departamento de Biblioteconomía y Documentación de la Universidad Complutense y accesible exclusivamente a través de Internet (http://multidoc.rediris.es/cdm).

Se trata en esta ocasión de un monográfico sobre Filmotecas, archivos fílmicos y medios de comunicación cinematográficos en la era digital: tendencias, que se propone reflejar la situación actual que presentan los ámbitos de la documentación y la investigación aplicados a la profesión y el trabajo cinematográficos, así como la evolución que dichos ámbitos están experimentando constantemente en función del propio desarrollo de la sociedad de la información y de las tecnologías digitales. Se ofrecerán las perspectivas, experiencias, aplicaciones y desarrollos realizados en universidades, instituciones de investigación y estudio, filmotecas, archivos fílmicos, medios de comunicación y asociaciones, fundaciones e instituciones y organismos especializados.

La invitación a colaborar en la gestación de dicho número fue cursada a IWETEL con fecha 6 de febrero de 2006, y asimismo solicitada a directores de filmotecas y archivos fílmicos, así como a responsables y documentalistas en servicios y centros de documentación de medios de comunicación cinematográficos y ámbitos cinematográficos de medios informativos en general.

\section{Otras actuaciones a emprender, otras propuestas de trabajo cinematográfico en red, otros proyectos}

Se requieren medidas para fomentar el cine y difundir su actividad en todos sus ámbitos, como algunos autores han puesto de manifiesto (Clavell, 2006). Hace falta un cambio en la legislación sobre propiedad intelectual que coordine de forma más coherente los derechos de autor y los otros derechos de la propiedad intelectual con el derecho colectivo a la cultura y el derecho de participación individual, reconocido en la Declaración Universal de Derechos del Hombre, para conseguir los siguientes objetivos:

- Hacer accesible al ciudadano en todo caso la obra audiovisual, articulando un sistema que permita, en caso de conflicto, salvaguardar los derechos de propiedad mediante el pago de un precio equitativo.

- Adoptar medidas que permitan a los titulares de derechos de propiedad su renuncia total o parcial de los derechos de explotación, temporal o definitiva.

- Revitalizar las filmotecas, dentro de este cambio legislativo, para que su principal cometido sea la difusión de las obras realizadas en el marco de su ámbito territorial.

Scire. 14 : 2 (jul.-dic. 2008) 41-64. ISSN 1135-3716. 
Para ello, es imprescindible dotarlas de medios técnicos y de personal suficientemente cualificado tanto para su cometido actual de organización de archivos como para la difusión de sus fondos mediante las nuevas tecnologías; facilitar el sistema que permita la obtención de copias de las obras archivadas; y proporcionar acceso on-line a todas las obras audiovisuales y cinematográficas de dominio público.

Por otra parte, sobre la normativa vigente en España en materia de depósito legal se constata que, pese a las variaciones sufridas a lo largo del tiempo en el ámbito legal, nunca se ha efectuado el depósito de películas. Para la salvaguarda y conservación del patrimonio cinematográfico resulta necesaria la inclusión del depósito de las películas cinematográficas en la legislación sobre el depósito legal (Oliván, 2000).

No obstante, en el momento de redactar estas páginas se acaba de aprobar en el Congreso de los Diputados (22 de junio de 2006) la reforma de la Ley de Propiedad Intelectual. Con un resultado que ha dejado satisfechas especialmente a las sociedades de derechos de autor, falta todavía aprobar la Ley de Cine y del Audiovisual.

En relación con proyectos de índole nacional y fundamentalmente internacional en los que podría o debería implicarse el ámbito cinematográfico, conviene hacer una breve referencia a los siguientes: RedIRIS2, EUMEDCONNECT, RedCLARA, TEIN2 y GÉANT2 (Robles, 2006). La actual infraestructura nacional, RedIRIS2, se clausura el 31 de octubre 2006; a partir de entonces se establecerá una nueva infraestructura de comunicaciones que pasará a denominarse RedIRIS10. En cuanto a proyectos internacionales relacionados con redes, la red EUMEDCONNECT se ha extendido todavía más, y se han creado enlaces troncales entre los nodos de EUMEDCONNECT y GÉANT2 que han aumentado a 622 Mbps. RedCLARA se ha ampliado ya a todos los países iberoamericanos, con las únicas excepciones de Honduras, Paraguay y Cuba.

La intranet mundial de la investigación se ha complementado con el despliegue de una red entre países de Asia y Australia llamada TEIN2 (Trans-Eurasia Information Network, www.tein2.net). El desarrollo de esta red pertenece a un proyecto financiado por la Comisión Europea cuyo objetivo es construir una infraestructura de interconexión entre las redes de investigación de los países del área Asia-Pacífico y la nueva estructura de red europea (GÉANT2). El nodo de GÉANT2 en España está situado en Madrid. Entre los días 28 de febrero y 1 de marzo de 2006 RedIRIS migró su conexión de GÉANT a GÉANT2.

\section{Conclusiones y recomendaciones}

Tras la finalización de este estudio, se destacan las siguientes conclusiones: 1) todavía no están todas las filmotecas españolas presentes en la red de redes; 2) las filmotecas presentes en Internet disponen, en general, de muy pocos contenidos, 
y en su mayor parte son meramente indicativos de lo que es la institución y de los servicios que presta; 3 ) solo se contabilizan dos o tres casos que disponen de mayor diversidad de materiales, independientemente de la información acerca de préstamos, catálogos de fondos, etcétera (relación de películas restauradas y otros documentos fílmicos), y en la mayoría de los casos son de pago; 4) a la accesibilidad de los fondos debería añadirse asimismo la gratuidad en su recuperación; 5) no existen casos de filmoteca y archivo fílmico, al menos conocidos por el autor, interconectados entre sí a través de Internet, aunque pueda haberlos en modo intranet.

A la vista de los resultados y de las conclusiones extraídas del trabajo, se plantean nuevos problemas y vías de estudio, resumidas en los estos puntos: 1) aunque se cuenta con algún precedente, muy tímido por cierto, deberían establecerse y generalizarse acuerdos de colaboración y proyectos conjuntos entre instituciones cinematográficas para la digitalización de sus fondos fílmicos; 2) dichos acuerdos y proyectos deberían extenderse también a instituciones no exclusivamente cinematográficas pero estrechamente relacionadas, tanto de ámbito público como privado; 3) deberían solicitarse apoyos en el marco de instituciones de investigación, tanto en el ámbito nacional como en el internacional — por ejemplo, Patrimonio.es o e Content Programme (estímulo al desarrollo de contenidos digitales europeos en redes mundiales y fomento de la diversidad ligüística en la sociedad de la información) - ; 4) para el conocimiento global y la difusión de la labor que llevan a cabo las filmotecas, y para contribuir al conocimiento de sus fondos documentales, deberían relacionarse con grandes redes académicas como, por ejemplo, la red latinoamericana ALICE, que interconecta las redes de investigación nacionales de América Latina a través de su sitio web RedCLARA; 5) asimismo, habría que implicarse de una forma u otra en actuaciones como la conformación de una biblioteca digital europea emprendida por la Unesco, e involucrarse también en el proyecto de biblioteca digital universal; dentro de dicho ámbito se debería incidir en la posible propuesta de inclusión, desarrollo y reconocimiento del término filmoteca digital universal; 6) el que los fondos documentales estén accesibles o de alguna forma consultables vía web gratuitamente o mediante pago podría paliar de algún modo o en parte los horarios restringidos de filmotecas y archivos fílmicos, pensando, claro está, en el trabajo que llevan a cabo investigadores, profesores, estudiantes, cinéfilos, comunicadores en general, etcétera.

Finalmente, se hace una propuesta de producción y difusión informativa colaborativa sobre el estado de la cuestión, mediante la invitación a colaborar en la publicación vía web de un monográfico sobre la temática planteada, en función de las informaciones y datos obtenidos como consecuencia de la realización del presente trabajo y de la metodología aplicada al mismo. Y, asimismo, una propuesta de digitalización de otros posibles fondos de filmotecas o de otros ámbitos —universitarios o no- estrechamente relacionados con el mundo de los archivos fílmicos. 


\section{Referencias}

Arencibia Jorge, Ricardo; Santillán Aldana, Julio; Subirats Coll, Inma (2005). Iniciativas de acceso abierto en ciencias de la información y documentación: evolución y perspectivas de E-LIS. // Revista Española de Documentación Científica. 28:2 (2005) 221-232.

CineDocNet: portal de documentación e investigación cinematográfica (2002-2006). Madrid: Servicio de Documentación Multimedia, Departamento de Biblioteconomía y Documentación, Facultad de Ciencias de la Información. http://multidoc.rediris.es/ descarga/cinedocnet, http://multidoc.rediris.es/cinedocnet (2006-06-10).

Clavell, Gaspar (2006). Reflexiones en torno a la divulgación de la obra cinematográfica. // Cuadernos de Documentación Multimedia. 16 (2005-2006). http://multidoc.rediris.es/ cdm/viewarticle.php?id=36\&layout=html (2006-06-13).

Cuadra de Colmenares, Elena de la (2006a). Filmotecas en línea. Propuesta de evaluación de páginas web de las filmotecas españolas. // Cuadernos de Documentación Multimedia. 16 (2005-2006). http://multidoc.rediris.es/cdm/viewarticle.php?id=33\&layout=html (2006-06-13).

Cuadra de Colmenares, Elena de la (2006b). British Pathé: análisis de la página web de un archivo fílmico. // Cuadernos de Documentación Multimedia. 16 (2005-2006) http://multidoc.rediris.es/cdm/viewarticle.php?id=37\&layout=html (2006-06-10).

Gil Urdiciain, Blanca; Pérez Agüera, José Ramón; Sánchez Jiménez, Rodrigo (2004). Gestión de documentación multimedia con Synchronized Multimedia Integration Language (SMIL). // Scire: Representación y Organización del Conocimento. 10:2 (jul.-dic. 2004) 87-97.

Gómez Romero, Amparo (2006). Análisis y evaluación del sitio web del Instituto Valenciano de Cinematografía. // Cuadernos de Documentación Multimedia. 16 (2005-2006). http://multidoc.rediris.es/cdm/viewarticle.php?id=38\&layout=html (2006-06-10).

Filmoteca de Catalunya (2001). Fons de Nitrats de la Filmoteca. Vol. I: Films de Ficció. Barcelona: Generalitat de Catalunya, 2001 (CD-ROM).

Filmoteca de Catalunya (2004). Fons de Nitrats de la Filmoteca. Vol. II: Films de no-ficció. (1896-1925). Barcelona: Generalitat de Catalunya, 2004 (CD-ROM).

Filmoteca de Catalunya (2005). Fons de Nitrats de la Filmoteca. Vol. III: Films de no-ficció (1926-1952). Barcelona: Generalitat de Catalunya, 2005 (CD-ROM).

López Yepes, Alfonso (2001). Cine y fuentes de información en Internet: panorámica documental y repertorio básico de sitios web. // Métodos de Información. 38-39 (2001) (CD-ROM y accesible vía web). http://multidoc.rediris.es/cinedocnet/textos/yepespdf.pdf (2006-06-05).

López Yepes, Alfonso (codir.) (2002). Fuentes del cine español en Internet (2002): Programa del Amo: proyecto de investigación en línea subvencionado por el Vicerrectorado de Relaciones Internacionales de la UCM y la Universidad Davis-California. http://www.ucm.es/info/multidoc/amoproyecto/default.html (2006-06-04).

López Yepes, Alfonso (dir.) (2003). Documentación cinematográfica: Mediateca. Proyecto de Innovación Educativa, financiado por el Vicerrectorado de Estudios de la Universidad Complutense. Madrid: Editorial Complutense (CD-ROM multimedia y accesible vía web) http://multidoc.rediris.es/mediateca (2006-06-15).

Scire. $14: 2$ (jul.-dic. 2008) 41-64. ISSN 1135-3716. 
López Yepes, Alfonso (2004). Redes temáticas: hacia una red iberoamericana de documentación informativa (InfoDocNet). // Jornadas Posicionamiento.es. El posicionamiento en Internet de instituciones culturales, científicas y educativas (Alicante, 5-7 mayo 2004) (CD-ROM). http://cervantesvirtual.com/posicionamiento (2006-06-02).

López Yepes, Alfonso; Pérez Agüera, José Ramón (2002). CineDocNet, TvDocNet: una comunidad virtual de usuarios de documentación audiovisual (cinematográfica y televisiva). // El Profesional de la Información. 11:5 (sep.-oct. 2002) (soporte impreso y CD-ROM).

López Yepes, Alfonso; Sánchez Jiménez, Rodrigo; Pérez Agüera, José Ramón (2003). Tratamiento de la documentación audio-visual en el entorno digital: iniciativas de metadatos y lenguajes de descripción multimedia.// El Profesional de la Información, 6, (nov.-dic. 2003) (soporte impreso y CD-ROM).

López Yepes, Alfonso; Sánchez Jiménez, Rodrigo; Pérez Agüera, José Ramón (2005a). Líneas de investigación y desarrollo tecnológico en el Departamento de Biblioteconomía y Documentación de la Universidad Complutense. // Documentación de las Ciencias de la Información. 28 (2005) 33-47.

López Yepes, Alfonso; Sánchez Jiménez, Rodrigo; Pérez Agüera, José Ramón (2005b). Agentes de información en entornos distribuidos y sistema multiagente de recuperación de información. // Investigación Bibliotecológica. 19:39 (jul.-dic. 2005) 28-46.

López Yepes, Alfonso (dir.); Cuadra Colmenares, Elena de la; Sánchez Jiménez, Rodrigo; Pérez Agüera, José Ramón (2006). El cine en la era digital: aplicaciones de la documentación cinematográfica (1992-2005). Madrid: Fragua, 2006 (incluye DVD).

Nistal, Ana (2006). Preservación digital en España: situación actual y perspectivas de futuro. // La preservación del patrimonio digital: conceptos básicos y principales iniciativas. Madrid: Ministerio de Cultura - Red.es, 16 de marzo de 2006. http://www.mcu.es/ bibliotecas/jornadas/jppd/ficheros/ana_nistal_jpd.ppt (2006-06-02).

Oliván Plazaola, Montserrat (2000). Patrimonio cultural, películas cinematográficas y depósito legal. // Boletín de la Asociación Andaluza de Bibliotecarios. 60 (2000). http://www.aab.es/pdfs/baab60/60a3.pdf (2006-06-03).

Robles, Esther (2006). Actualidad y proyectos de red: RedIRIS2. Conexiones externas. Proyectos internacionales para la creación de redes: EUMEDCONNECT, RedCLARA y TEIN2, GÉANT2. // Boletín de RedIRIS. 76 (abril 2006) 3-5. http://www.rediris.es/ rediris/boletin/76/actualidad.pdf (2006-06-05).

Salvador Benítez, Antonia; Ruiz Rodríguez, Antonio Ángel (2006). Metadatos para la preservación de contenidos digitales. // Cuadernos de Documentación Multimedia. 16 (2005-2006). http://multidoc.rediris.es/cdm/viewarticle.php?id=35\&layout=html (200606-13).

Torrado Morales, Susana (2006). Análisis comparativo de las web de las filmotecas españolas en Internet. // Cuadernos de Documentación Multimedia. 16 (2005-2006). http://multidoc.rediris.es/cdm/viewarticle.php?id=34\&layout=html (2006-06-03). 